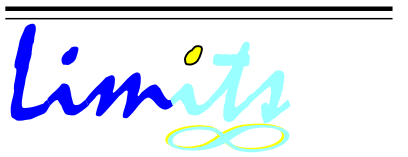

J. Math. and Its Appl.

ISSN : 1829-605X

Vol. 2, No. 2, Nov 2005, 61-69

\title{
Some Known Results and an Open Problem of Tree - Wheel Graph Ramsey Numbers
}

\author{
Surahmat \\ Department of Mathematics Education \\ Universitas Islam Malang \\ Jalan MT Haryono 193, Malang 65144,Indonesia \\ caksurahmat@yahoo.com
}

\begin{abstract}
There are many famous problems on finding a regular substructure in a sufficiently large combinatorial structure, one of them i.e. Ramsey numbers. In this paper we list some known results and an open problem on graph Ramsey numbers. In the special cases, we list to determine graph Ramsey numbers for trees versus wheels.
\end{abstract}

Keywords: Ramsey number, tree, wheel

\section{Introduction}

Before we present about graph Ramsey numbers, we rewrite the story of classical Ramsey numbers in [26] as follows.

"The origins of Ramsey theory are diffuse. Frank Plumton Ramsey [23] was interested in decision procedures for logical systems. Issai Schur wanted to solve Fermats last theorem over finite fields. B.L. van der Waerden solved an amusing problem and immediately returned to his researches in algebraic geometry. The 
emergence of Ramsey theory as a cohesive subdiscipline of combinatorial analysis occurred only in the last decade, see [14].

Issai Schur[25] proved the first theorem of what was later to be called Ramsey theory in 1916. He proved that: For every $r \in \mathcal{N}$ there exists an $n \in \mathcal{N}$ such that, given an arbitrary $r$-coloring of $S=\{1,2, \ldots, n\}$; there exist $x, y, z \in S$ all the same color, satisfying $x+y=z$. His motivation for establishing this result was the study of Fermats Last Theorem over finite fields. In the 1920s he made the following conjecture: If the positive integers are divided into two classes, at least one of the classes contains an arithmetic progression of $\mathrm{k}$ terms, no matter how large the given length $\mathrm{k}$ is. Over lunch one day in 1926, B. L. van der Waerden told Emil Artin and Otto Schreier about this problem. Immediately after lunch they went into Artins office in the Mathematics Department of the University of Hamburg and tried to find a proof. They solved the question of Schurs conjecture and it was later formally proved by Van der Waerden. Ramsey proved his famous theorem in 1930 in the first 8 pages of a 20 page paper On a problem of formal logic [23]. Ramseys theorem may be stated as follows: Let $k, r, n$ be positive integers. If $N$ is sufficiently large and if the $k$-sets of an $N$-set are colored arbitrarily with $r$ colors then there exists an $n$-set, all of whose $k$ element subsets are the same color. Ramsey needed this result for his researches in Mathematical Logic and he used this theorem to establish a result in a decision procedure for a certain class of statements in First Order Logic. It is ironic that it was discovered later that Ramseys theorem was not needed for constructing the required decision procedure. This happened during the Hilbert-program, which attempted to find a general decision procedure for statements in First Order Logic. What is even more ironic is that Kurt Godels[19] undecidability results (which were published the year after Ramsey died) showed that such a decision procedure could not exist. Thus Ramsey theory is named after Frank Plumpton Ramsey because he proved a theorem he did not need, in the course of trying to do something we now know cannot be done! The proof of Van der Waerdens theorem made a great impression on a young mathematician named Richard Rado. He may be considered the first true Ramsey theoretician, since in his $\mathrm{PhD}$ dissertation (under the supervision of Issai Schur) and in his subsequent work he was interested in Ramsey theory problems per se. Ramseys theorem was rediscovered in the classic 1935 paper [11] of Paul Erdos and George Szekeres. Erdos and Szekeres were young students in Budapest at the time and one of their friends in Budapest, Esther Klein, discovered that: given any 5 points in a plane, some four points form a convex quadrilateral. They soon made a general conjecture: for any $\delta$ there exists an $\epsilon$ so that given $\epsilon$ points in the plane, some $\delta$ form a convex set. Szekeres wrote in the foreword of [10]:

I have no clear recollection how the generalization actually came about; 
in the paper we attributed it to Esther, but she assures me that Paul had much more to do with it. We soon realized that a simple minded argument would not do and there was a feeling of excitement that a new type of geometric problem emerged from our circle which we were only too eager to solve. For me, [the] fact that it came from Epszi (Pauls nickname for Esther, short for epsilon) added a strong incentive to be the first with a solution and after a few weeks I was able to confront Paul with a triumphant E.P., open your wise mind. What I had really found was Ramseys Theorem, from which [the above result] easily followed. Of course, at that time none of us knew about Ramsey.

It is believed that what we now know as Ramsey theory went into a long embryonic stage from 1930 to 1973 and that it was really born at the Combinatorial Conference at Balatonfured, Hungary during 1973. The conference proceedings [20] reveal that there were more than 24 talks devoted to what is now called Ramsey theory. Among the speakers were Richard Rado, Walter Deuber, Klaus Leeb, Ron Graham, and Paul Erdos in whose honour the conference was held. Ramsey theory found its place as a cohesive sub-discipline of combinatorial analysis at the Balatonfured conference and is concerned with conditions that guarantee that a combinatorial object necessarily contains some smaller given objects. The least number of sub-objects that guarantees the existence of some smaller objects is called a Ramsey number. Therefore the role of Ramsey numbers is to quantify some of the general existential theorems in Ramsey theory. The first Ramsey number was published as a result of the 1953 Putnam competition. Leo Mozer phoned Frank Harary from Edmonton asking for a graphical problem which would complete the Putnam competition which he was composing. He suggested the following problem of which the solution and commentary is given by Gleason, Greenwood and Kelly [17] in their comprehensive review and commentary on these collected problems and solutions:

Problem. Six points are in a general position in space (no three in a line, no four in a plane). The fifteen line segments joining them in pairs are drawn and then painted, some segments red, some blue. Prove that some triangle has all its sides the same color.

Solution. Let $\mathrm{P}$ be any of the six points. Five of the line segments end at P, and of these at least three, say PQ, PR and PS, must have the same color, say blue. Then, if any one of the segments QR, RS and $\mathrm{SQ}$ is blue we will have a blue triangle, and if not, QRS will be a red triangle. Thus in any event at least one triangle has all its sides the same color.

The above mentioned problem is a part of the famous party problem: What is 
the fewest number of people at a birthday party that will guarantee three mutual acquaintances or three mutual strangers? The answer is 6 people. Greenwood and Gleason first published this result (which is considered the first publication of a non-trivial Ramsey number) in the Canadian Journal of Mathematics in 1955 [16].

This subject has grown tremendously, in particular with regard to asymptotic bounds for various types of Ramsey numbers. The progress on evaluating the basic numbers themselves has been very unsatisfactory for a long time.

Nextly, generalized of classical of Ramsey numbers i.e. graph Ramsey numbers, considerable progress has been made new area Ramsey theory. In this paper, we determine the graph Ramsey numbers especially cycle-wheel.

\section{Basic concept}

In this paper, all graphs are finite and simple. Let $G$ be such a graph. We write $V(G)$ or $V$ for the vertex set of $G$ and $E(G)$ or $E$ for the edge set of $G$. The graph $\bar{G}$ is the complement of the graph $G$, i.e., the graph obtained from the complete graph $K_{|V(G)|}$ on $|V(G)|$ vertices by deleting the edges of $G$.

The graph $H=\left(V^{\prime}, E^{\prime}\right)$ is a subgraph of $G=(V, E)$ if $V^{\prime} \subseteq V$ and $E^{\prime} \subseteq E$. For $x \in V$ and a subgraph $B$ of $G$, define $N_{B}(x)=\{y \in V(B): x y \in E\}$ and $N_{B}[x]=N_{B}(x) \cup\{x\}$. The degree $d_{G}(x)$ of a vertex $x$ is $\left|N_{G}(x)\right|$.

$C_{n}$ be a cycle of $n$ vertices. A tree $T_{n}$ be a connected graph of $n$ vertices containing no a cycle as a subgraph. A walk of length $n$ from a vertex $u$ to a vertex $v$ in $G$ is sequence of vertices $\left(u=u_{o}, u_{1}, \ldots, u_{n}=v\right)$ such that $u_{i-1} u_{i} \in E(G)$ for each $i$. A closed walk has $u_{0}=u_{n}$. A path is walk in which all are distinct. A Star $S_{n}$ is a graph whose a center $x$ adjacent to all other $n-1$ vertices of degree one. $W_{m}=\{x\}+C_{m}$ be a wheel with a rim $V\left(C_{m}\right)=\left\{x_{1}, x_{2}, \ldots, x_{m}\right\}$ and a hub $x$.

For given graphs $G$ and $H$, the graph Ramsey number $R(G, H)$ is the smallest positive integer $N$ such that for every graph $F$ of order $N$ the following holds: either $F$ contains $G$ as a subgraph or the complement of $F$ contains $H$ as a subgraph. If $G$ is a complete graph $K_{a}$ and $H$ is also a complete graph $K_{b}$, we usually write $R(a, b)$ and be called Classical Ramsey numbers. In the following section, we present some known result of classical Ramsey numbers and graph Ramsey numbers.

\section{Some results on classical Ramsey numbers}

Some fundamental results on classical Ramsey numbers $R(a, b)$, we will rewrite in below. 
Lemma 3.1 For any $b \geq 2, R(1, b)=1$ and $R(2, b)=b=R(b, 2)$.

Lemma 3.2 For any $a, b \in \mathcal{N}, R(a, b)=R(b, a)$.

Lemma 3.3 (Erdös and Szekeres[11]). For $a, b \geq 3, R(a, b) \geq(a-1)(b-1)+1$ and $R(a, b) \leq R(a-1, b)+R(a, b-1)$ with strict inequality if both $R(a-1, b)$ and $R(a, b-1)$ are even.

For small cases, we have only known nine exact value of classical Ramsey numbers and the other only upper bound and lower bound which are difference with Lemma 3 i.e.:

Tabel of Small Classical Ramsey numbers $R(a, b)$

\begin{tabular}{|c|c|c|c|c|c|c|c|c|c|c|c|}
\hline $\begin{array}{c}\mathrm{a} \\
\mathrm{b}\end{array}$ & 3 & 4 & 5 & 6 & 7 & 8 & 9 & 10 & 11 & 12 & 13 \\
\hline 3 & 6 & 9 & 14 & 18 & 23 & 28 & 36 & 43 & 51 & 59 & 69 \\
& & & & & & & & 40 & 46 & 52 & 59 \\
\hline 4 & & 18 & 25 & 41 & 61 & 84 & 115 & 149 & 191 & 238 & 291 \\
& & & & 35 & 49 & 56 & 69 & 92 & 97 & 128 & 133 \\
\hline 5 & & & 49 & 87 & 143 & 216 & 316 & 442 & & & \\
& & & 43 & 58 & 80 & 101 & 121 & 141 & 157 & 181 & 205 \\
\hline 6 & & & & 165 & 298 & 495 & 780 & 1171 & & & \\
& & & & 102 & 111 & 127 & 169 & 178 & 253 & 262 & 317 \\
\hline 7 & & & & & 540 & 1031 & 1713 & 2826 & & & \\
& & & & & 205 & 216 & 232 & & 405 & 416 & 511 \\
\hline 8 & & & & & & 1870 & 3583 & 6090 & & & \\
& & & & & & 282 & 317 & & & & 817 \\
\hline
\end{tabular}

\section{Some results on graph Ramsey numbers}

There has been more activity and considerably more results in graph Ramsey numbers than in classical Ramsey numbers. It would be impossible to take all even a fraction of the results, so we will review $R(G, H)$ just a few of the highlights.

For $G$ and $H$ are a path $P_{m}$ and $P_{n}$, respectively, Geréncser and Gyárfas [13] found the Ramsey in the following theorem.

Theorem 4.1 For positive integers $n \geq m \geq 2, R\left(P_{m}, P_{n}\right)=n+\left\lfloor\frac{m}{2}\right\rfloor-1$.

Faudree and Schelp [12] and Rosta [24] obtained the graph Ramsey numbers for combination cycles and cycles in below. 


\section{Theorem 4.2}

$R\left(C_{n}, C_{m}\right)=\left\{\begin{array}{l}2 n-1 \text { for } 3 \leq m \leq n, m \text { odd },(n, m) \neq(3,3) . \\ n+\frac{m}{2}-1 \text { for } 4 \leq m \leq n, m \text { even and } n \text { even, }(n, m) \neq(4,4) . \\ \max \left\{n+\frac{m}{2}-1,2 m-1\right\} \text { for } 4 \leq m<n, m \text { even and } n \text { odd. }\end{array}\right.$

Chvátal and Harary [7] found the result by concept of chromatic number and the largest of component in the following theorem.

Theorem 4.3 $R(G, H) \geq(\chi(G)-1)(s(H)-1)+1$, where $s(H)$ is the number of vertices of the largest component of $H$ and $\chi(G)$ is the chromatic number of $G$.

For combination of a complete graph $K_{m}$ and a tree $T_{n}$ Chvátal[8] obtained the result:

Theorem 4.4 For integer $m, n \geq 1, R\left(K_{m}, T_{n}\right)=(m-1)(n-1)+1$.

Bondy and Erdos got the graph Ramsey numbers for combinations a complete graph $K_{m}$ and a cycle $C_{n}$ :

Theorem 4.5 If $m \geq 3$ and $n \geq m^{2}-2$, then $R\left(K_{m}, C_{n}\right)=(m-1)(n-1)+1$.

Since 1976, It was conjecture that $R\left(K_{m}, C_{n}\right)=(m-1)(n-1)+1$ for $m \geq$ $n \geq 3$, except $m=n=3$. For more information, see nice survey small Ramsey numbers in [21].

\section{Some known results on tree-wheel}

For combination of tree and wheel, several results have been obtained for wheels. For instance, Surahmat and E.T. Baskoro[27, 1] obtained the Ramsey numbers of Path $P_{n}$ versus wheels $W_{m}$ as follows:

Theorem $5.1 R\left(P_{n}, W_{m}\right)=2 n-1$ if $m=4$ and $n \geq 4$ or $m \geq 6$ is even and $n \geq \frac{m}{2}(m-1)+1$.

For combination a star and a wheel, see in [27] and [28], we obtained:

Theorem $5.2 R\left(S_{n}, W_{4}\right)=2 n-1$ if $n \geq 3$ odd or $R\left(S_{n}, W_{4}\right)=2 n+1$ if $n \geq 4$ even. 
Theorem 5.3 For all $n \geq 2 m-4, m \geq 5$ and $m$ odd, $R\left(S_{n}, W_{m}\right)=3 n-2$

To Determine of the Ramsey Graph numbers of any tree $T_{n} \neq S_{n}$ versus $W_{m}$ for $n \geq m-1$, we have information from [2] as below:

Theorem 5.4 Let $n \geq 4$ and assume that we are given a particular tree $T_{n}$ of $n$ vertices other than a star. Then the Ramsey number $R\left(T_{n}, W_{4}\right)=2 n-1$.

Theorem 5.5 Let $n \geq 3$ and assume that we are given a particular tree $T_{n}$ of $n$ vertices other than a star. Then the Ramsey number $R\left(T_{n}, W_{5}\right)=3 n-2$.

\section{Open Problem}

In this section we shall give in the following an open problem:

Determine the Ramsey numbers of Tress versus Wheels in general?

\section{References}

[1] E.T. Baskoro and Surahmat, The Ramsey number of paths with respect to wheels, Discrete Mathematics 294 (2005) 275-277.

[2] E. T. Baskoro, Surahmat, S.M. Nababan, and M. Miller, On Ramsey graph numbers for all trees versus $W_{4}$ or $W_{5}$, to appear in Graphs and Combinatorics (2002).

[3] J. A. Bondy, Pancyclic graphs, J. of Combinatorial Theory Ser. B 11 (1971) 80-84.

[4] S. Brandt, R.J. Faudree and W. Goddard, Weakly pancyclic graphs, J. of Graph Theory 27 (1998) 141-176.

[5] S. A. Burr and P. Erdős, Generalization of a Ramsey-theoretic result of Chvátal, J. of Graph Theory 7 (1983) 39-51.

[6] V. Chvátal and P. Erdős, A note on Hamiltonian circuits, Discrete Math. 2 (1972) 111-113.

[7] V. Chvátal and F. Harary, Generalized Ramsey theory for graphs, III. Small off-diagonal numbers, Pacific J. of Math. 41 (1972) 335-345.

[8] V. Chvátal, Tree-complete graph Ramsey numbers, Journal Graph Theory 7 (1977) 93. 
[9] G. Dirac, Some theorems on abstract graphs, Proc. London Math. Soc. 2 (1952) 69-81.

[10] P. Erdos, The Art of Counting, (J.H. Spencer ed.), MIT Press, Cambridge, $M A,(1973)$.

[11] P. Erdös and G. Szekeres, A combinatorial problem in geometry, Compositio Math. 2 (1935) 463-470.

[12] R. J. Faudree and R. H. Schelp, All Ramsey numbers for cycles in graphs, Discrete Mathematics 8 (1974) 313-329.

[13] L. Geréncser and A. Gyárfas, On Ramey-type problems, Ann. Univ.Sci. Budapest Eötvös Sect. Math 10 (1967) 167-170.

[14] R.L. Graham, B.L. Rothschild and J.H. Spencer, Ramsey Theory, John Wiley and Sons, New York, (1990).

[15] J.E. Graver and J. Yackel, Some Graph Theoretic Results Associated with Ramseys Theorem, Journal of Combinatorial Theory, 4 (1968), 125175.

[16] A.M. Gleason and R.E. Greenwood, Combinatorial Relations and Chromatic Graphs, Canadian Journal of Mathematics, 7 (1955), 17.

[17] A.M. Gleason, R.E. Greenwood and L.M. Kelly, The Putnam Mathematical Competition, Problems and solutions: 19381964, Math. Assoc. Amer., Washington, (1980), 365366.

[18] U. Grenda and H. Harborth, The Ramsey number $r(K 3 ; K 7-e)$, Journal of Combinatorics, Information-System Sciences, 7 (1982), 166-169.

[19] K. Godel, Uber formal unentscheidbare Satze der Principia Mathematica und verwander Systeme I, Monatshefte fur Mathematic und Physik, 38 (1931), 173198.

[20] (A. Hajnal, R. Rado and V.T. Sos eds.), Infinite and finite Sets, North Holland, New York, (1975).

[21] S. P. Radziszowski, Small Ramsey numbers, Electronic J. of Combinatorics (2004) DS1.8.

[22] S. P. Radziszowski and J. Xia, Paths, cycles and wheels without antitriangles, Australasian J. of Combinatorics 9 (1994) 221-232.

[23] F.P. Ramsey, On a problem of formal logic,Proc. London Math. Soc. 30 (1930) 264-286. 
[24] V. Rosta, On a Ramsey type problem of J.A. Bondy and P. Erdős, I \& II, J. of Combinatorial Theory (B) 15 (1973) 94-120.

[25] I. Schur, Uber die kongruenz $\mathrm{xm}+\mathrm{ym}=\mathrm{zm}(\bmod \mathrm{p})$, Jber. Deutsch Math. Verein., 25 (1916), 114116

[26] E. H. Stipp, Bounds for Ramsey numbers in multipartite graphs, Preprint (2000).

[27] Surahmat and E.T. Baskoro, On the Ramsey number of a path or a star versus $W_{4}$ or $W_{5}$, Proceedings of the 12-th Australasian Workshop on Combinatorial Algorithms, Bandung, Indonesia, July 14-17 (2001) 174-179.

[28] Surahmat, E.T. Baskoro and H.J. Broersma, The Ramsey number of large star-like trees versus large odd wheels, Memorandum, 1621, University of Twente The Netherlands, (2002). 